\title{
Implantes zigomáticos de carga imediata: relato de caso
}

\author{
Immediate load zygomatic implants: case report
}

\author{
Sérgio Ricardo Almeida1*, Danilo Barral Araújo², Elisângela de Jesus Campos ${ }^{3}$, Gabriela Botelho Martins ${ }^{4}$ \\ ${ }^{1}$ Professor do Núcleo Innovare de Educação em Saúde, Salvador, Bahia; 2,4 Professor Associado e Docente do \\ Programa de Pós-graduação Processos Interativos dos Órgãos e Sistemas. ICS - UFBA; ${ }^{3}$ Professor Adjunto e Docente \\ do Programa de Pós-graduação Processos Interativos dos Órgãos e Sistemas. ICS - UFBA
}

\begin{abstract}
Resumo
Introdução: atualmente, o relevante desafio enfrentado pela odontologia se constitui na redução do tempo de tratamento e na restrição dos procedimentos cirúrgicos, na perspectiva de se assegurar o máximo conforto às pessoas que buscam a reabilitação oral. Em se tratando de pacientes edêntulos totais, os eventos que devem ser desenvolvidos demandam de um planejamento correto tendo em consideração o tempo de execução. Face a esses pressupostos, a reabilitação oral busca reconstituir a mastigação, a estética, o suporte labial e a fonética. Objetivo: o caso clínico ora descrito visa demonstrar a instalação de 4 implantes zigomáticos seguindo-se da colocação da prótese definitiva em sistema de carga imediata. Metodologia: com base na literatura científica e tendo em vista a limitação das condições anatômicas e clínicas apresentadas pela paciente do sexo feminino, com 68 anos de idade e boa saúde, fez-se a opção pela instalação de 4 implantes zigomáticos associada à técnica de carga imediata. Resultado e conclusão: em compatibilidade com os dados da literatura, o êxito cirúrgico e a reabilitação oral alcançados, associados à satisfação manifestada pela paciente, são indicadores de elevada previsibilidade quanto à longevidade da sobrevida dos implantes zigomáticos instalados.
\end{abstract}

Palavras-chave: Osso zigomático. Arcada edêntula. Maxila. Carga imediata em implante dentário.

\begin{abstract}
Introduction: currently, the relevant challenge faced by dentistry constitutes in reduce treatment time and restrict surgical procedures, in order to ensure maximum comfort to people seeking oral rehabilitation. In case of total patient treatment, the events to be displayed require correct planning, taking into account the execution time. Do to these assumptions, the oral rehabilitation seeks to reconstitutes chewing, aesthetics, lip and phonetic support. Objective: the clinical case aims to demonstrate the installation of 4 zygomatic implants following the placement of the definitive prosthesis in the immediate loading system. Methodology: based on scientific literature and in view of the limitation of the anatomical and clinical conditions applied by the 68-year-old female patient, she choose to install 4 zygomatic implants used in the immediate loading technique. Result and conclusion: in line with literature data, surgical and oral rehabilitation achieved, associated with patient satisfaction, are indicators of increased predictability regarding the longevity of survival of the zygomatic implants used.
\end{abstract}

Keywords: Zygoma, Jaw. Edentulous. Maxilla. Immediate dental implant loading.

\section{INTRODUÇÃO}

A reabilitação de pacientes edêntulos totais é um procedimento complexo, uma vez que envolve entre outros fatores, a reconstituição das funções primárias de mastigação, da estética, do suporte labial e da fonética. Por outro lado, os eventos essenciais à reabilitação oral demandam de um planejamento correto tendo em consideração o tempo de execução. Assim sendo se constitui num desafio a redução desse tempo frente à complexidade dos procedimentos de caráter invasivo que se impõem. Diante isso, o relevante desafio da odontologia atual é reduzir o tempo de tratamento e minimizar os eventos cirúrgicos, dando assim mais conforto ao paciente. ${ }^{1-3}$

Correspondente/Corresponding: *Sérgio Ricardo Matos de Almeida - Núcleo Innovare de Educação em Saúde. - End resid.: Rua Thomaz Gonzaga 246 (casa 5). Salvador, BA. CEP: 41.110-050. Tel: (38) 988950459. - E-mail: sralmeida40@gmail.com
Aspectos relevantes da reabilitação oral podem ser abordados com o uso de implantes osseointegrados, uma abordagem alternativa que resulta na forma, na função e na estética satisfatória assegurada pelas unidades dentárias. Todavia, o sucesso desse tipo de prótese cirúrgica para a reabilitação oral requer o cuidado adequado com a estrutura óssea que irá suportar e ancorar os dispositivos dentários para que os impactos da mastigação possam ser absorvidos, portanto, bem tolerados. ${ }^{4,5}$

Devido a certas situações excepcionais que podem ocorrer na cavidade oral, como a periodontite, a perda de dentes, as extrações traumáticas, os cistos, os tumores ou os traumas agravados ao longo do tempo, levam o cirurgião nem sempre a encontrar as condições mais favoráveis para realizar o tratamento de reabilitação envolvendo a instalação de implantes. ${ }^{6,7}$

Há que se ter em conta que maxila apresenta características que podem trazer dificuldades à reabilitação, como a pneumatização dos seios maxilares, a presença 
do forame incisivo e fossas nasais além, é claro, da própria atrofia severa do processo alveolar, o que dificulta a retenção e estabilidade das próteses totais, promovendo alteração da dimensão vertical, da fala e da mastigação, além de comprometer a harmonia facial e por consequência, a autoestima dos indivíduos. 2,3,8,9

Para recuperar as dimensões ideais de tamanho perdidas pelos ossos alveolares que sofreram reabsorção, particularmente, nos pacientes desdentados, é necessário aumentar a altura e a largura da estrutura óssea para acomodar os implantes de tamanho apropriado com um ângulo axial que permita, adequadamente, a instalação da prótese futura. ${ }^{8,10}$

Objetivando superar essas dificuldades enfrentadas no processo de reabilitação oral, os implantes zigomáticos (ZIS) surgiram como um método alternativo desde o ano de 1998, concebido pelo Professor Branemark. Tal feito representou uma excelente alternativa para as situações de reabilitação, inicialmente, nas vítimas de traumas e/ou cirurgias ressectivas de tumores, onde grandes porções ósseas são removidas. De acordo com Duarte, Peredo e Melo ${ }^{11}$, é indicada a utilização de fixações zigomáticas em sistema de carga imediata, pois a esplintagem rígida dos implantes inclinados distribui as cargas axiais e laterais fazendo com que haja estabilidade do sistema reabilitador.

A osseointegração de implantes dentários é considerada hoje em dia, altamente previsível. No entanto, a reabilitação de maxilas atróficas extremas e totalmente desdentadas por meio da inserção de implantes padrão ainda é muito difícil, mesmo com a realização de procedimentos de enxertia óssea. ${ }^{1-3,12}$ Para esses casos, os implantes zigomáticos (ZIS) surgiram como um método alternativo para ultrapassar as situações supracitadas. ${ }^{8,10}$

Face a estes aspectos, os implantes convencionais tiveram sua geometria modificada, viabilizando sua instalação. Recomenda-se nos dias atuais, inclusive, a realização de carga imediata, pois a esplintagem rígida dos implantes inclinados distribui as cargas axiais e laterais, fazendo com que haja estabilidade do sistema reabilitador.

Durante as duas últimas décadas, a colocação de implantes zigomáticos, normalmente inseridos através do seio maxilar, tendo sua porção apical fixada e estabilizada no osso zigomático, tem provado ser uma opção eficaz na reabilitação da maxila desdentada atrófica grave. Implantes zigomáticos se constituem numa opção viável nas maxilas atróficas, uma vez que tornam possível a obtenção de um excelente travamento primário no processo de reabilitação oral sem a necessidade dos enxertos ósseos que são mais comumente empregados. ${ }^{13}$

É importante se observar que estudos realizados desde 1997 demonstram um percentual de sucesso superior à $95 \%$. Este panorama é fortalecido associando-se as fixações zigomáticas (FZ's) à carga imediata, diminuindo substancialmente, o tempo de reabilitação dos pacientes, independente da etiologia. ${ }^{11,12}$
As fixações zigomáticas são implantes de grande comprimento integrados em dois pontos no osso zigomático resultando em duas possibilidades de reabilitação no rebordo, sendo que a primeira necessita de pelo menos dois implantes anteriores associados a dois implantes zigomáticos, podendo ser ativados tardiamente ou imediatamente. A segunda possibilidade, é a utilização de quatro implantes zigomáticos sem a utilização de implantes anteriores de preferência, sendo ativados imediatamente, formando um polígono fechado para melhor distribuição das cargas mastigatórias. ${ }^{1,11,14-16}$

Esta alternativa suprime a necessidade de enxerto ósseo em pacientes com reabsorção maxilar reconhecidamente moderada ou severa, objetivando simplificar o tratamento com a possibilidade de devolver de forma imediata a função, aumentar a previsibilidade dos resultados, diminuir os riscos, os custos econômicos, biológicos e a morbidade, se constituindo numa alternativa clínica para os procedimentos reconstrutivos. ${ }^{2,16,17}$

\section{RELATO DE CASO}

Paciente do sexo feminino, com 68 anos de idade e boa saúde, buscou tratamento odontológico referindo que utilizava prótese total superior há mais de 20 anos, tendo expressado alto grau de insatisfação com os sons produzidos pela sua fala em decorrência da precariedade de retenção da prótese total superior em uso. Relatou já ter passado por procedimento cirúrgico prévio visando reabilitar a arcada superior na qual fora realizada cirurgia pré-protética de aprofundamento do vestíbulo com a intenção de melhorar a retenção da prótese total mucossuportada. No decorrer da anamnese, a paciente atribuiu o insucesso às limitações da região anatômica, tendo exposto, ainda, ter sido submetida a tratamento cirúrgico recente para instalação de prótese inferior mediante a opção pelo protocolo "all on four" que consiste em um tipo de prótese implantossuportada que possui uma barra metálica fixada em quatro implantes osseointegrados instalados na região interforames mentonianos, atuando como pilares para toda a estrutura metaloplástica da prótese. $O$ fato de ter obtido êxito na reabilitação inferior com a adoção desta técnica, desejou que a mesma opção reabilitadora fosse utilizada na arcada superior.

Ao ser avaliada a tomografia da maxila da paciente (Figura 1) foi constatado que não havia altura, tampouco volume ósseo suficiente para receber implantes convencionais. Para tanto, foi analisada a possibilidade de aplicação da técnica sinus-lift bilateral, por se tratar de um tipo de procedimento indicado para condicionar a região a receber os implantes, uma vez que se trata de uma opção compensatória da altura e do volume de osso basal. Contudo, a paciente não aceitou ser submetida ao mencionado procedimento, uma vez que desejava ser reabilitada mais rapidamente e com o menor número de intervenções cirúrgicas possíveis. 
Através do exame de imagem obtido por tomografia computadorizada foi constatada uma reabsorção generalizada dos processos alveolares da maxila, assim como, extensa pneumatização do seio maxilar, bilateralmente (Figura 2).

Figura 1 - Tomografia inicial de maxila

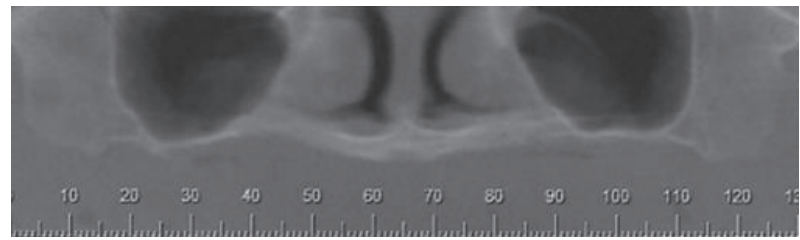

Fonte: Autoria própria

Figura 2 - Tomografia de seios e fossas nasais

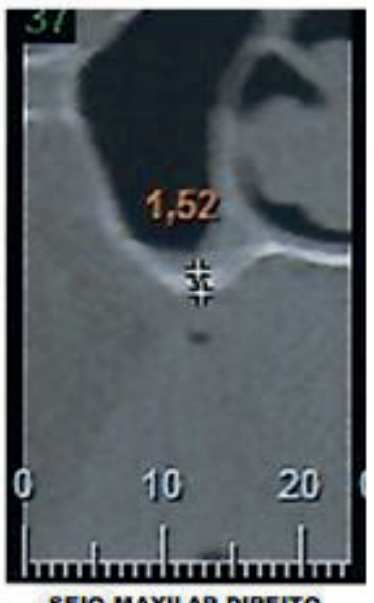

SEIO MAXILAR DIREITO

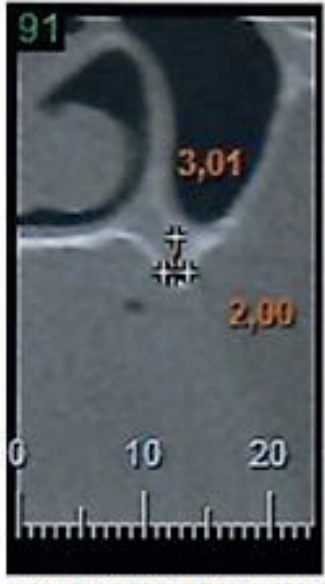

SEIO MAXILAR ESQUERDO

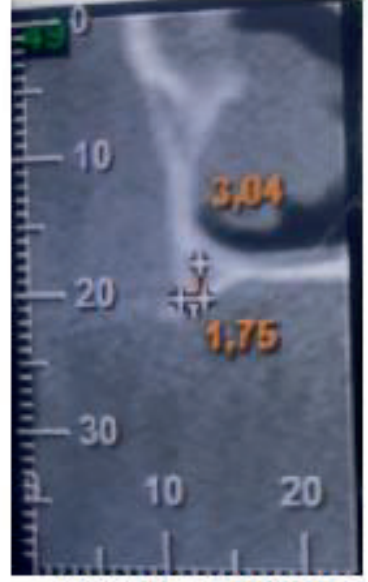

FOSSA NASAL DIREITA

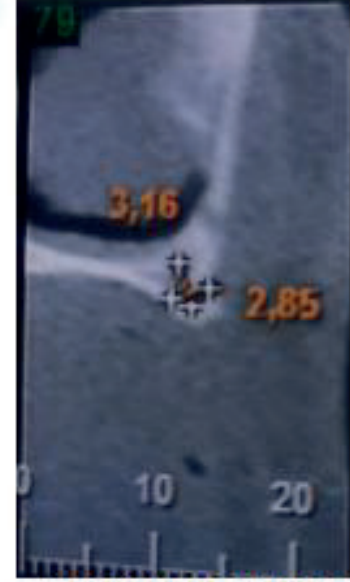

FOSSA NASAL ESQUERDA
Fonte: Autoria própria
Frente a essa situação clínica e a recusa da paciente em se submeter aos vários procedimentos cirúrgicos necessários à reconstrução maxilar, a fim de viabilizar a instalação de implantes convencionais, foi proposta como alternativa para a resolução do caso, a instalação de 4 implantes zigomáticos, por se tratar de um recurso de baixo custo, menor morbidade, alta taxa de sucesso, com alta previsibilidade e menor tempo de reabilitação para a paciente, de acordo com Duarte, Peredo e Melo ${ }^{11}$. As fixações zigomáticas viabilizam a reabilitação sem a necessidade de grande enxertos ósseos, extensas feridas cirúrgicas e longos períodos de pós-operatório. ${ }^{18}$

Antes da realização do procedimento cirúrgico, foram realizados procedimentos protéticos para a confecção de um guia multifuncional (Figura 3), utilizado-o tanto no procedimento cirúrgico para orientação do correto posicionamento dos implantes como nos procedimentos protéticos de moldagem de transferência. Para o ato cirúrgico realizado em ambiente hospitalar sob anestesia geral, foram requisitados os exames e consultas com os
As quantidade ósseas apresentadas pela paciente na região de base dos seios maxilares e das fossas nasais regiões nas quais são comumente fixados os implantes convencionais - no que diz respeito à altura, conforme pode ser constatado na Figura 2, não foi constatado volume ósseo suficiente para realizar a anconragem dos implantes. Ao exame tomográfico foi mensurado uma altura óssea de $1,52 \mathrm{~mm}$ no assoalho de seio maxilar direito, $3,01 \mathrm{~mm}$ em assoalo de seio maxilar esquerdo, $3,04 \mathrm{~mm}$ em fossa nasal direita e $3,16 \mathrm{~mm}$ em fossa nasal esquerda. Estas medidas inviabilizaram, completamente, a instalação de implantes convencionais sem que se lançasse mão de técnicas adjuvantes como o levantamento de membrana de seio maxilar, bilateralmente, aumentando, sobremaneira, a morbidade para a paciente e, sobretudo, o tempo para a realizar a reabilitação com implantes para posterior instalação da prótese.
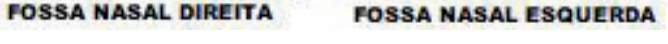

especialistas visando assegurar a boa condição sistêmica da paciente confirmando, portanto, sua condição de paciente ASA I.

Figura 3 - Guia multifuncional

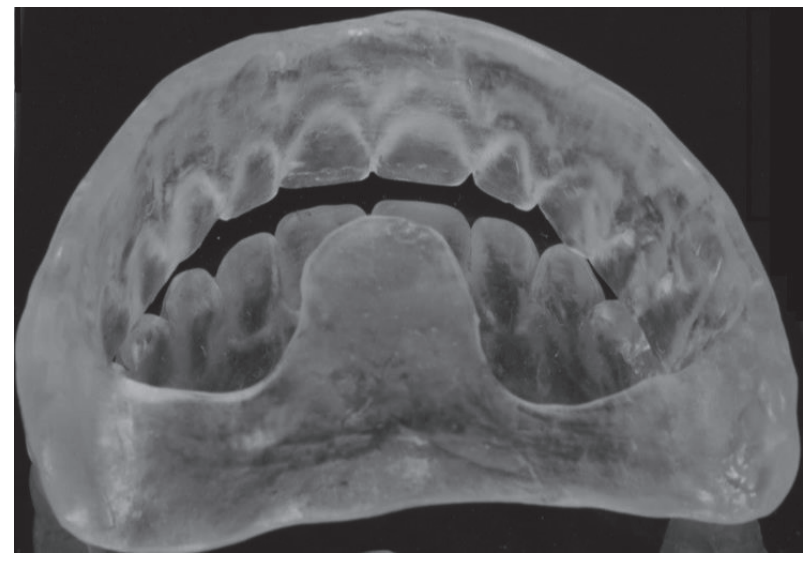

Fonte: Autoria própria 
Após a anestesia geral, foi iniciado o procedimento cirúrgico que consistiu, preliminarmente, na realização de infiltração anestésica com vasoconstrictor, utilizado tubetes de 1,8 $\mathrm{mL}$ com solução anestésica de articaína a 4\% e epinefrina 1:100.000 em toda a região vestibular da maxila e do palato com o objetivo de reduzir o sangramento. A incisão foi realizada na crista do rebordo maxilar estendendo de uma tuberosidade à contralateral, e de duas incisões verticais relaxantes de $1 \mathrm{~cm}$ na extensão posterior. A seguir, toda a extensão da maxila foi exposta até a abertura piriforme, pilares caninos, zigomáticos e todo o processo alveolar (Figura 4).

Figura 4 - Acesso cirúrgico

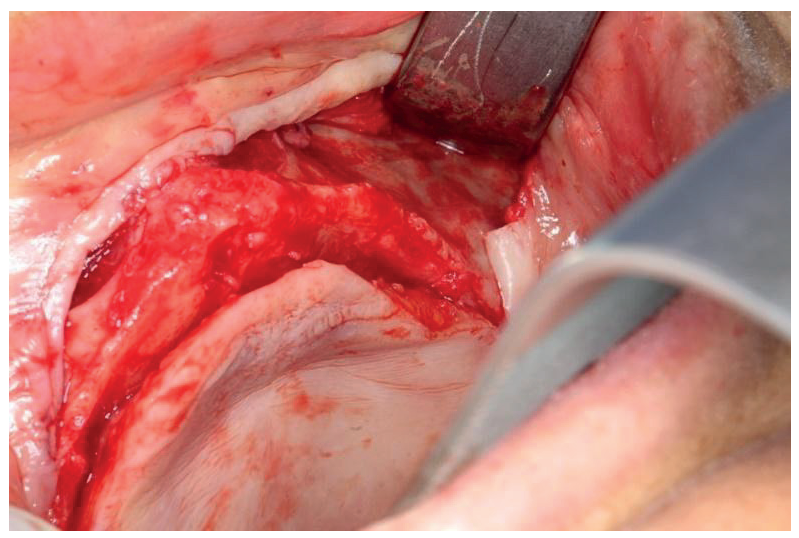

Fonte: Autoria própria

Para a instalação dos implantes foi a Técnica da Ranhura Lateral desenvolvida por Stella e Warner em 2000, uma vez que se trata de uma técnica que possibilita a orientação mais vertical para o acesso ao osso zigomático e elimina a necessidade de uma abertura sinusal, diminuindo assim a morbidade do procedimento cirúrgico. A instalação dos 4 implantes $\operatorname{SIN}^{\circledR}$ foi feita de tal forma, que os implantes com tamanhos maiores $(4,5 \times 42,5 \mathrm{~mm}$ e $4,5 \times 52,5 \mathrm{~mm}$ ) foram posicionados mais distalmente no corpo do zigoma em região infra-orbitária, tendo a plataforma protética emergindo na região do primeiro molar esquerdo e direito, respectivamente, enquanto que os implantes menores (4,5 x 40mm e 4,5 x 35mm) foram fixados mais anteriormente ao osso zigomático, tendo sua plataforma emergindo na região de caninos e incisivos laterais, direito e esquerdo, respectivamente. 0 torque obtido de travamento ósseo dos implantes foi em média $45 \mathrm{~N}$, proporcionando, inclusive, uma boa estabilidade primária, condição que viabiliza com segurança, o procedimento de carga imediata. Ao se observar a Figura 5 , pode-se constatar a configuração obtida pós-instalação dos implantes.

\section{Figura 5 - Organização espacial dos implantes} instalados

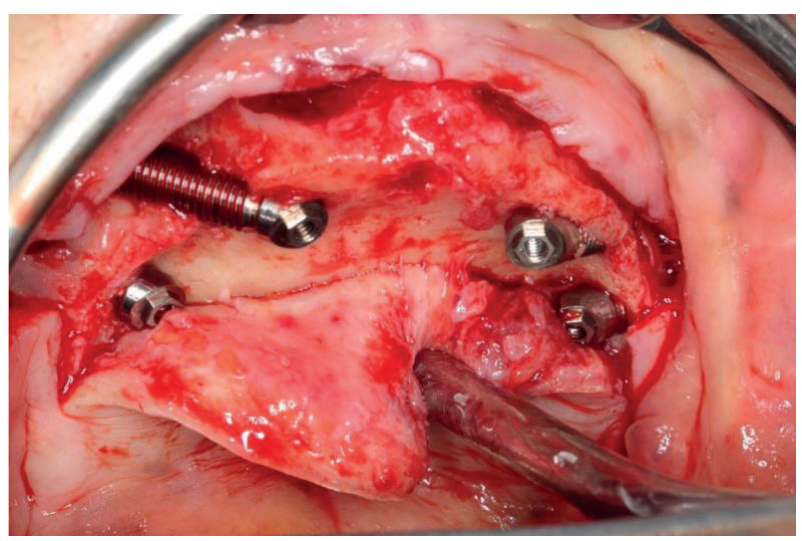

Fonte: Autoria própria

Na sequência do próprio ato cirúrgico foram conectados os pilares protéticos aplicados com torque de $20 \mathrm{~N}$, seguido-se da instalação das tampas de proteção. Dando continuidade, foi realizada uma incisão no palato no local de emergência do pilares para o reposicionamento do retalho e posterior sutura com fio reabsorvível, Vicryl ${ }^{\circledR}$ 4.0 (Figura 6).

Figura 6 - Cirurgia finalizada com instalação de abutments e tampas de proteção

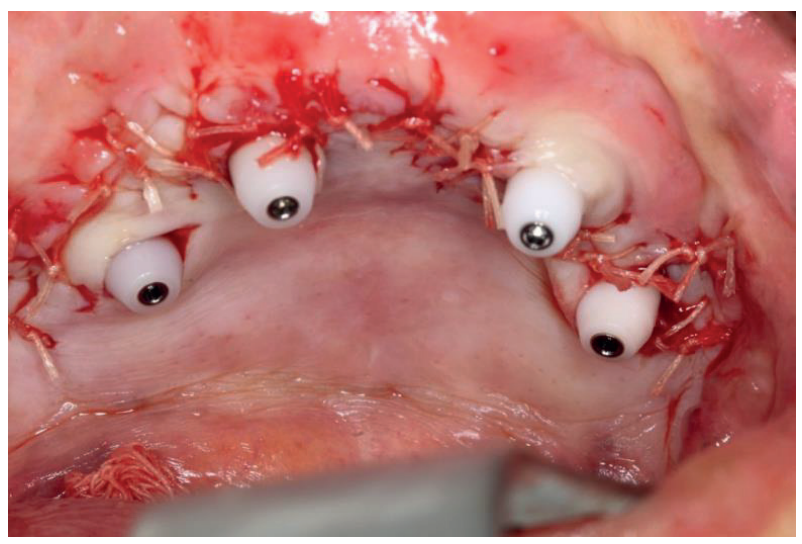

Fonte: Autoria própria

O trabalho protético foi iniciado em ambulatório no dia seguinte à cirurgia. Foram removidas as tampas de proteção e instalados os componentes protéticos para a transferência com o guia multifuncional (Figura 7) 
Figura 7- Moldagem de transferência

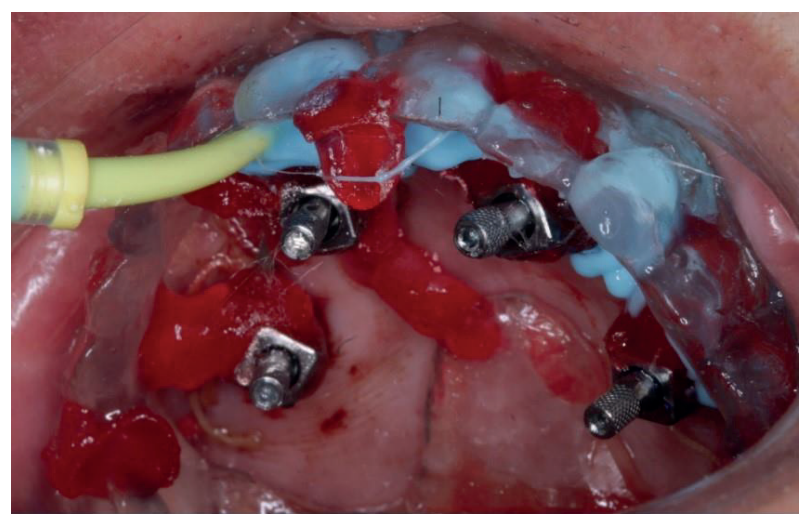

Fonte: Autoria própria

Realizados os procedimentos técnico-laboratoriais de acrilização, pode ser instalada a prótese definitiva (Figura 8) três dias após o ato cirúrgico sucedida da realização dos primeiros ajustes oclusais e das orientações de higienização da prótese.

Figura 8 - Prótese instalada e ajuste oclusal sendo realizado

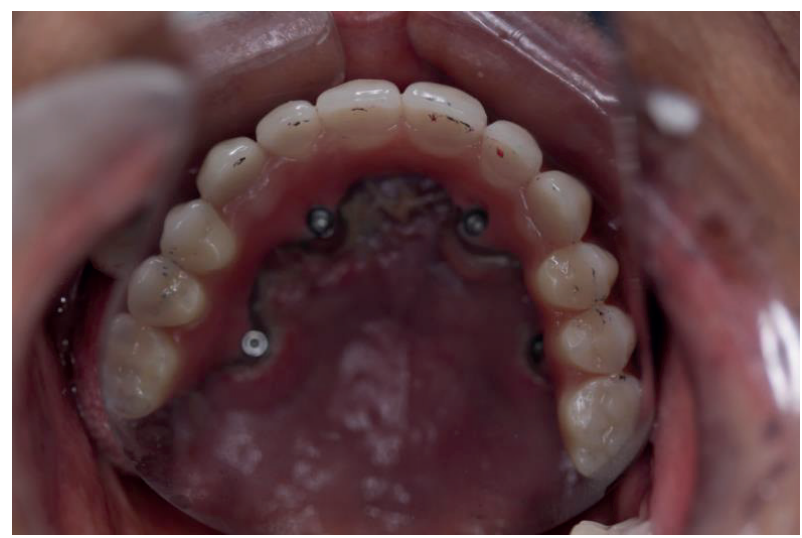

Fonte: Autoria própria

Após a instalação da prótese, a paciente retornou para controle e foram solicitados os seguintes exames radiográficos: panorâmica, projeção anterior de seio maxilar (Figura 9) e Waters (Figura 10). Por três semanas consecutivas foram feitos os ajustes oclusais considerados necessários, sendo então liberada a paciente e orientada ao retorno ambulatorial uma vez por mês durante os seis primeiros meses.
Figura 9-Imagem radiográfica dos implantes e barras metálicas

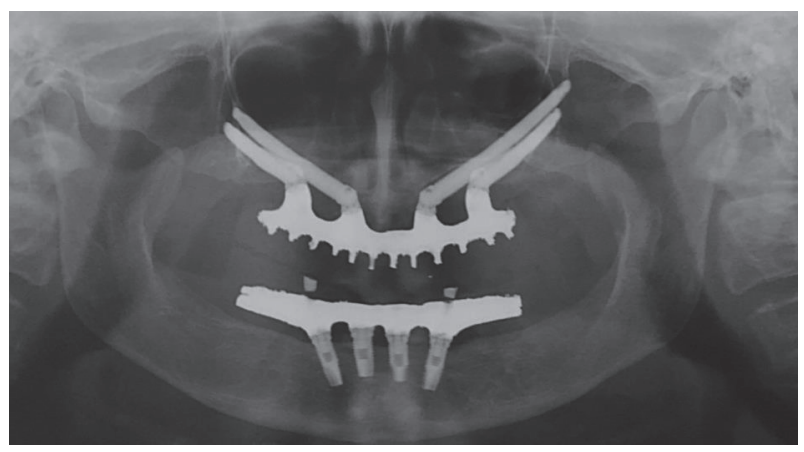

Fonte: Autoria própria

Figura 10 - Imagem radiográfica dos implantes e barras metálicas, técnica de Waters

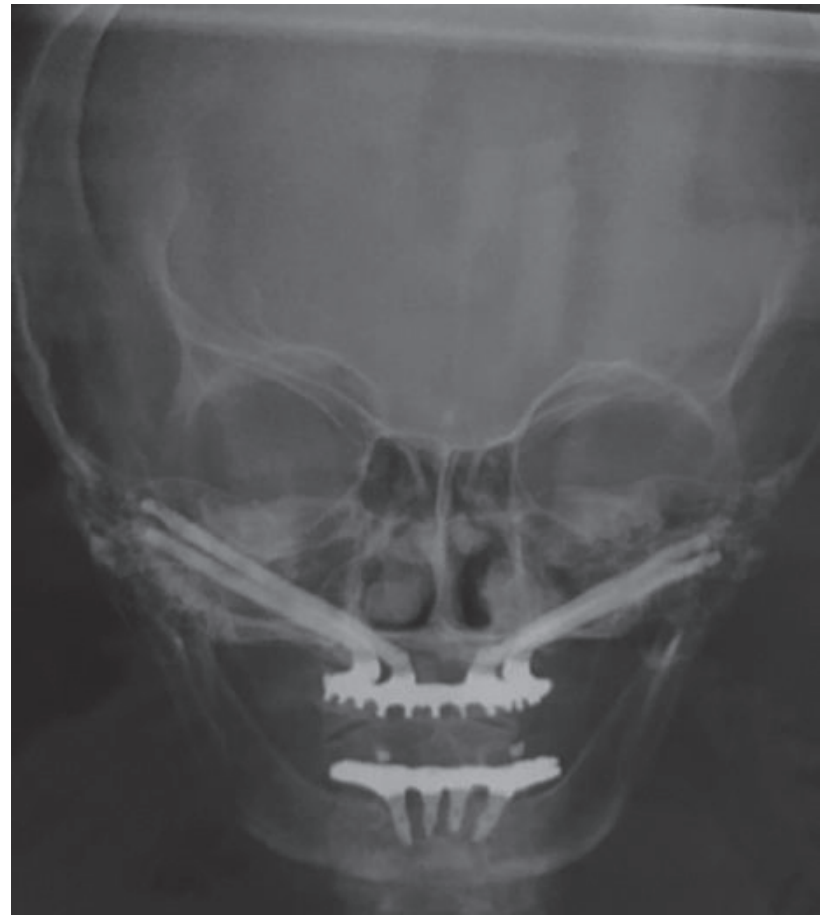

Fonte: Autoria própria

\section{RESULTADO E CONCLUSÃO}

Pacientes que apresentam maxilas atróficas, ou não têm rebordo alveolar suficientemente volumoso. Esta característica implica, muitas vezes, na limitação das condições essenciais à estabilização de próteses totais convencionais. De igual forma, aqueles que apresentam seio maxilar se estendendo anteriormente para a cavidade nasal, também possuem redução da quantidade de osso para a ancoragem de implantes convencionais. Tais situações representam um desafio clínico terapêutico para ser realizada a reabilitação protética com implantes convencionais. ${ }^{9,13,19}$

Branemark propôs duas classificações dos estágios de reabsorção da maxila. A primeira na direção antero-posterior, ocorre um estreitamento da maxila que parece estar em posição posterior a mandíbula. A segunda classificação 
se refere à configuração do seio maxilar e à evolução da pneumatização proporcional ao grau de atrofia, conforme Duarte, Peredo e Melo ${ }^{11}$. A segunda classificação de A a E, se refere à configuração do seio maxilar e a evolução da pneumatização proporcional ao grau de atrofia. Segundo Sutton et al. ${ }^{20}$, uma reabilitação cirúrgica e protética de pacientes edêntulos objetiva a restauração da função oral e da forma facial. O planejamento do tratamento requer um entendimento dos efeitos da atrofia progressiva dos maxilares e concomitante efeito sobre os tecidos moles da face. ${ }^{11,15}$

Após avaliações detalhadas com base na mencionada classificação descrita por Branemark, pode-se identificar que a paciente de que trata o presente caso clínico apresentava um severo grau de reabsorção maxilar, reunindo as características indicativas de que implantes zigomáticos estariam melhor indicados para a sua reabilitação.

Em que pese Branemark preconizar a técnica que consistia em abrir uma janela na parede lateral do seio maxilar, descolar sua membrana e em seguida, inserir os implantes zigomáticos através do mencionado seio maxilar até sua fixação no osso zigomático ${ }^{11,21}$, outras técnicas foram surgindo ou adaptando a técnica original de Branemark visando diminuir a morbidade, torná-la menos invasiva, se adaptar às variações anatômicas da região ou, até mesmo, ampliar a penetração do implante zigomático no osso malar, melhorando, dessa forma, a estabiliadade primária. Técnicas como a de Stella e Warner ${ }^{22}$ que ao invés de abrir uma janela na parede do seio, criava somente, uma pequena abertura na parede dessa estrutura biológica a fim de visualizar a passagem do implante pelo seio maxilar até a sua inserção no zigoma. ${ }^{1,11,14,21}$

Outra variação da técnica de Branemark foi desenvolvida também, com o intuito de reduzir a morbidade e favorecer uma melhor distribuição das plataformas protéticas dos implantes zigomáticos, permitindo com esta metodologia, a confeção de próteses melhor adaptadas anatomicamente. Trata-se da Técnica Externalizada ou Extra-sinual, que consiste em modificar a angulação de fixação do implante zigomático mantendo-o fora do seio maxilar, deixando a maior parte do corpo do implante em contato com o tecido mole, sendo ancorado somente, no osso malar. ${ }^{21}$

Portanto, em face das características clínicas encontradas e das limitações anatômicas da paciente, no que diz respeito a volume ósseo em região do seio maxilar eno formato da arcada, fez-se a opção pela Técnica de Stella e Warner. ${ }^{22}$

Existem muitos fatores que interferem no resultado final no que diz respeito às próteses fixas na quase totalidade dos pacientes com maxila atrófica severa. Durante um longo tempo os procedimentos de enxertia, antes ou durante a instalação dos implantes, tornaram-se rotineiros. Várias técnicas de aumento e regeneração óssea estão descritos na literatura científica, todos com o objetivo comum de reestabelecer o arcabouço ósseo para a instalação dos implantes convencionais. Entretanto, o alto custo, a alta morbidade e possíveis incidências de falhas, com alguma frequência se sobressaem aos resultados favoráveis. Estas ponderações foram levadas em consideração na seleção da técnica que foi empregada no caso em estudo. ${ }^{13,14,16}$

Os implantes zigomáticos foram desenvolvidos nos primórdios para reabilitar pacientes maxilectomizados, vítimas de traumas ou de cirurgias ressectivas. Com o passar dos anos foram sendo utilizados em cirurgias reabilitadoras de maxilas atróficas a fim de reduzir a morbidade dos procedimentos cirúrgicos, reduzir custos e acelerar a reabilitação protética do paciente. ${ }^{2,14,16}$

Estudos de longevidade demonstram que a sobrevida e a taxa de êxito dos implantes zigomáticos quando utilizados para reabilitação de maxilas severamente atrofiadas se situa entre 3 e 10 anos de instalados, apontando, portanto, taxas de sucesso em torno de 90 a $97 \%{ }^{15,16}$. Em contraste com estes achados, estudo descrito por Salgado $^{16}$ registra uma taxa de sucesso referente à colocação de implantes convencionais em pacientes previamente sujeitos a elevação do seio maxilar correspondente a $74 \%$, considerada a instalação entre 3 e 5 anos. ${ }^{16}$

Os implantes zigomáticos têm como uma das características principais suportar vários tipos de restaurações protéticas podendo ser, cimentadas, parafusadas ou overdenture ${ }^{16}$. Portanto, a reabilitação utilizando implantes zigomáticos se apresenta como favorável para este tipo de paciente uma vez que viabiliza a reabilitação num curto espaço de tempo. Este pressuposto de redução do tempo de reabilitação tem sido observado, consensualmente, entre os autores, conforme a vasta literatura científica que aborda o conceito de carga imediata. ${ }^{1,14,16}$

Em virtude da alta taxa de êxito que, por sinal, se situa em torno de $90 \%$ com base no que foi anteriormente discutido, a opção pela técnica de carga imediata demonstrada pode ser recomendada como forma de reabilitar mais rapidamente em poucos dias, o paciente, utilizando-se, apenas, um procedimento cirúrgico, tal como acha-se explicitado no presente estudo de caso.

\section{REFERÊNCIAS}

1. KAWAKAMI, P. Y. et al. Implantes zigomáticos: revisão de literatura com estudo do índice de sucesso através de meta-análise. Rev. odontol. Univ. Cid. São Paulo, São Paulo, v. 23, n.1, p. 51-58, jan./abr. 2011.

2. OLIVEIRA, G. J. P. L. de. et al. Oral Rehabilitation with zygomatic implants in a patient with cleft palate. Case Rep. Dent., Bethesda, v. 2019, 5 p., 2019. DOI: 10.1155/2019/6591256

3. BALAN, I. et al. Treatment of severe trophic maxilla with zygomatic implants: a case series. Oral Implantol., Rome, v. 10, n. 3, p. 317-324, 2017.

4. ARAÚJO FILHO, N. C. de. New bone formation in maxillary sinuses of monkeys elevated and with grafted with hydroxyapatite and platelet-rich plasma thesis. 2001. 85f. Tese [Doutorado em Odontologia] - BBO, Rio de Janeiro, 2001.

5. GONÇALVES, A. R. Q. The association of platelet-rich plasma with inorganic bovine bone in maxillary sinus grafts induces new bone formation (monograph)?. Rio de Janeiro, 2008. p. 78. 
6. LINDHE, J.; KARRING, T.; LANG, N. P. Tratado de periodontia clínica e implantologia oral. 4. ed. Rio de Janeiro: Guanabara Koogan, 2005. p. 1-1013.

7. BUSER, D.; DAHLIN, C.; SCHENK, R. K. Guided bone regeneration in implant dentistry. Chicago: Quintessence, 1994. p. 1-265.

8. SCARANO, A. et al. Maxillary sinus augmentation with different biomaterials: a comparative histologic and histomorphometric study in man. Implant. Dent., Baltimore, v. 15, n.2, p. 197-207, 2006.

9. BASTOS, L. C. M. Reabilitação de maxilas atróficas com ancoragem zigomática: relato de casos clínicos. 2017. Faculdade Avantis, Salvador-Ba, 2017.

10. DALAPICULA, S. S. et al. Characteristics physicochemical of the biomaterials used of bone grafts: a critical review. ImplantNewsPerio, São Paulo, v.3, n. 5, p. 487-491, 2006.

11. DUARTE, L. R. da.; PEREDO, L. G.; MELO, G. F. de. Fixação zigomáticas na reabilitação de maxilas atróficas - estágio cientifico atual e pespectivas futuras. 2005. $6 p$.

12. BUTTERWORTH, C. J.; ROGERS, S. N. The zygomatic implant perforated (ZIP) flap: a new technique for combined surgical reconstruction and rapid fixed dental rehabilitation following low-level maxillectomy. Int. J. Implant. Dent., Baltimore, v. 3, n. 37, 2017. DOI: 10.1186/s40729017-0100-8.

13. GRECCHI, F. et al. A new surgical and technical approach in zygomatic implantology. Oral implantol., Abingdon, v. 10, n.2, p. 197-208, 2017.

14. CAMPANHA, B. P.; SILVA, A. L. Implantes zigomáticos: uma revisão de literatura. 2011. 71f. Monografia (Especialização) - Universidade Federal de Minas Gerais - Faculdade de Odontologia, Minas Gerais, 2011.

15. COELHO, T. M. K.; EGAMI, G. F. Avaliação radiográfica das fixações zigomáticas pela técnica exteriorizada modificada. 2015. 45f. Dissertação (Mestrado) - Universidade Federal de Mato Grosso do Sul, Campo Grande, 2015.

16. SALGADO, D. J. R. Reabilitação com implantes de maxila atrófica - implantes zigomáticos VS Implantes curtos - revisão de literatura. 2012. 35f. Faculdade de Medicina Dentária, Universidade do Porto, Portugal, 2012.

17. PINEAU, M. et al. L'implant zygomatique dans notre pratique quotidienne. Partie II: réhabilitation prothétique et influence sur la qualité de vie. Swiss Dent. J., Bern, v. 128, n. 9, p. 683-700, 2018.

18. MALAQUIAS, P. D. T. I. A. et al. Reabilitação de maxilla atrófica com implantes zigomáticos: relato de caso. Arch. Health Invest., Araçatuba, v. 2, n. 4. p. 29-34, 2013

19. WU, Y. et al. Eye-lid approach for four zigomatic implant placement in the severely reabsorbed maxillae: technical note. Int. J. Clin. Exp. Med., Madison, v. 8, n.3, p. 4670-4675, 2015.

20. SUTTON, D. N. et al. Changes in facial form relative to progressive atrophy of the edentulous jaws. Int. J. Oral Maxillofac. Surg., Copenhagen, v. 33, p. 676-682. 2004.

21. PATROCÍNIO, M. N.; CERQUEIRA, A. Fixações zigomáticas no tratamento das maxilas atróficas e suas complicações: uma revisão de literatura. Trabalho de Conclusão de Curso [Monografia] - Escola Bahiana de Medicina e Saúde Pública, Salvador/BA, 2012.

22. STELLA, J. P.; WARNER, M. R. Sinus slot technique for simplification and improved orientation of zygomaticus dental implants: a technical note. Int. J. Oral Maxillofac. Implants., Lombard, v. 15, p. 889-893, 2000.

Submetido em: 04/10/2019

Aceito em: 07/10/2019 